\title{
Electrocardiography-gated computed tomography angiography analysis of cardiac pulsatility-induced motion and deformation after endovascular aneurysm sealing with chimney grafts
}

\author{
Maaike A. Koenrades, PhD, ${ }^{a, c, d}$ Esmé J. Donselaar, MD, ${ }^{e, f}$ Mirthe A. J. M. van Erp, MSc, ${ }^{b}$ \\ Tom G. J. Loonen, MSc, ${ }^{b}$ Pim van Lochem, MSc, ${ }^{b}$ Almar Klein, PhD, ${ }^{g}$ Robert H. Geelkerken, MD, PhD, a,d and \\ Michel M. P. J. Reijnen, MD, PhD, ${ }^{a, e}$ Enschede, Arnhem, and Nieuwegein, The Netherlands
}

\begin{abstract}
Objective: To evaluate the proximal stability of the chimney endovascular aneurysm sealing configuration (chEVAS) during the cardiac cycle by investigating the cardiac pulsatility-induced movement and deformation.

Methods: We retrospectively analyzed postoperative electrocardiogram-gated computed tomography angiography scans of 11 chEVAS cases (9 primary chEVAS plus 2 chEVAS-in-chEVAS). ChEVAS procedures were conducted between September 2013 and June 2016. Motion and deformation of the EVAS stents, the chimney grafts, and the stented branch vessels were evaluated during the cardiac cycle using an established combination of image registration and segmentation techniques.

Results: Electrocardiogram-gated computed tomography angiography scans of 11 chEVAS configurations including 22 EVAS stents and 20 chimney grafts were analyzed. The three-dimensional displacement was at most $1.7 \mathrm{~mm}$ for both the EVAS stents and the chimney grafts. The maximum change in distance between components was no more than $0.4 \mathrm{~mm}$ and did not differ between EVAS-to-EVAS stent and EVAS stent-to-chimney stent $(0.2 \pm 0.1 \mathrm{~mm} \mathrm{vs} 0.2 \pm 0.1 \mathrm{~mm}$; $P=.823$ ). The mean change in chimney deflection angle was $1.2 \pm 0.7^{\circ}$; the maximum change was greatest for the superior mesenteric artery (SMA) $\left(2.6^{\circ}\right)$. The EVAS stent-to-chimney angles for the left renal artery, right renal artery, and SMA varied on average by $0.7 \pm 0.3^{\circ}$ (range, $0.4^{\circ}-1.3^{\circ}$ ), $1.0 \pm 0.3^{\circ}$ (range, $0.5^{\circ}-1.7^{\circ}$ ), and $0.8^{ \pm} 0.4^{\circ}\left(\right.$ range, $0.3^{\circ}-1.3^{\circ}$ ), respectively, during the cardiac cycle. The end-stent angles for the left renal artery, right renal artery, and SMA varied on average by $1.7 \pm 0.9^{\circ}\left(\right.$ range, $\left.0.5^{\circ}-3.3^{\circ}\right), 1.9 \pm 0.8^{\circ}\left(\right.$ range, $\left.0.7^{\circ}-3.3^{\circ}\right)$, and $1.3 \pm 0.4^{\circ}\left(\right.$ range $\left.0.7^{\circ}-1.6^{\circ}\right)$, respectively, during the cardiac cycle. Overall, the end-stent angles varied on average by $1.7 \pm 0.8^{\circ}$ (range, $0.5^{\circ}-3.3^{\circ}$ ).

Conclusions: The chEVAS configuration proved to be stable during the cardiac cycle, as demonstrated by minimal cyclical changes in distance between device components and angulation between the EVAS stents and the chimney grafts. The limited deflection angles of the chimney grafts decrease the risk of bending fatigue, but the more apparent change in end-stent angle distal to the chimney graft may raise concerns regarding late branch occlusion or stenosis. (J Vasc Surg 2020; :1-10.)
\end{abstract}

Keywords: Endovascular aneurysm sealing; Chimney grafts; Pulsatile motion; Electrocardiogram-gated computed tomography angiography; Proximal sealing and fixation

The use of chimney, or parallel, grafts combined with an endovascular aneurysm device provides a versatile alternative to fenestrated endografts for endovascular repair of pararenal and juxtarenal abdominal aortic aneurysms (AAA). ${ }^{1,2}$ Although the chimney technique in combination with conventional endografts may be troubled by

From the Multimodality Medical Imaging M3i Group, Faculty of Science and Technology, ${ }^{a}$ Technical Medicine, Faculty of Science and Technology, ${ }^{b}$ and Robotics and Mechatronics Group, Faculty of Electrical Engineering, Mathematics and Computer Science, ${ }^{c}$ Technical Medical Centre, University of Twente, Enschede: the Department of Vascular Surgery, Medisch Spectrum Twente, Enschede ${ }^{d}$; the Department of Vascular Surgery, Rijnstate, Arnhem ${ }^{e}$; the Department of Anesthesiology, St. Antonius Hospital, Nieuwegeinf; and the Independent Scholar, Enschede. ${ }^{9}$

Supported in part by an unrestricted grant from Endologix Inc. and in part by the PPP Allowance made available by Health Holland, Top Sector Life Sciences \& Health, to stimulate public-private partnerships.

Author conflict of interest: RG is consultant for Terumo Aortic, MR is consultant for Endologix, Bentley Innomed and Terumo Aortic. chimney graft compression and gutter-endoleak formation, $^{3}$ the combination with sac-sealing endografts (chEVAS) has the potential to prevent these complications. ${ }^{4,5}$ The multicenter ASCEND registry demonstrated favorable results for chEVAS, ${ }^{4}$ although the data are not robust beyond 12 months of follow-up. In addition,

Additional material for this article may be found online at www.jvascsurg.org. Correspondence: Maaike A. Koenrades, PhD, Multimodality Medical Imaging M3i Group, Faculty of Science and Technology, Technical Medical Centre, University of Twente, PO Box 217, 7500 AE Enschede, The Netherlands (e-mail: m.a.koenrades@utwente.nl).

The editors and reviewers of this article have no relevant financial relationships to disclose per the JVS policy that requires reviewers to decline review of any manuscript for which they may have a conflict of interest. 0741-5214

Copyright (c) 2020 by the Society for Vascular Surgery. Published by Elsevier Inc https://doi.org/10.1016/j.jvs.2020.01.064 
chEVAS was recently shown to be an effective method to treat complications of previous endovascular aneurysm repair procedures. ${ }^{6,7}$ However, endovascular aneurysm sealing (EVAS) is a relatively new technology, lacking long-term data, and concerns have been raised regarding the long-term outcome and failure modes, particularly caudal migration. ${ }^{8}$ The long-term stability of the endograft depends on its ability to withstand the repetitive stresses posed by the blood flow. Previous research has demonstrated that the degree of endograft motion may differ per fixation site and between devices, ${ }^{9}$ which underlines the importance to understand the individual device dynamics to allow for adequate durability tests. To achieve long-term stability, it seems mandatory that the individual stents and chimney grafts move as a single unit. However, no study has investigated this in a clinical setting. One may hypothesize that ongoing movements in stents might lead to stent fractures in time. In addition, one could also imagine that these chronic (micro)movements might lead to a loss of branch vessel patency as a result of the bending motion of the stented, vessel leading to tissue injury in the transition zone to the native vessel. This study focuses on the cardiac pulsatility-induced movement and deformation of the Nellix EVAS System (Endologix, Irvine, Calif) in combination with chimney grafts to evaluate its proximal stability during the cardiac cycle.

\section{METHODS}

Patient population. This study retrospectively analyzed electrocardiogram (ECG)-gated computed tomography angiography (CTA) scans that were acquired during the postoperative period after chEVAS procedures conducted in a Dutch AAA vascular center between September 2013 and June 2016. The ECG-gated CTA scans were performed at the discretion of the treating physician, mostly to enhance experience with the ECG-gated CTA technique. In these patients, the regular follow-up CTA scan was replaced by an ECG-gated CTA. Eleven cases were available for analysis. Nine patients (mean age, $77 \pm$ 6 years; eight men) underwent a primary chEVAS procedure; one of these with a Nellix-in-Nellix extension to treat failed EVAS owing to caudal stent migration with a type la endoleak. Postoperative ECG-gated CTA scans were available at a mean follow-up of $7.7 \pm 6.2$ months (range, 0-16 months). A redo chEVAS procedure (chEVAS-in-chEVAS procedure) was conducted for two of these patients at 20 and 26 months after primary chEVAS owing to a type la endoleak with aneurysm growth and a 7-mm migration of the Nellix stents and aneurysm growth without an evident endoleak but with a reduced seal owing to neck dilatation and a 2-mm downward migration of the Nellix stents. ${ }^{10}$ Both patients were treated with a triple chimney procedure by proximal extension of the primary unilateral chEVAS device configuration. Postoperative ECG-gated CTA scans were

\section{ARTICLE HIGHLIGHTS}

Type of Research: Retrospective, cohort study Key Findings: Analysis of 11 postchimney endovascular aneurysm sealing configuration electrocardiogramgated computed tomography angiography scans demonstrated minimal pulsatile motion and deformation of device components during the cardiac cycle.

Take Home Message: Endovascular aneurysm sealing combined with chimney grafts imparted a stable configuration during the cardiac cycle.

Table I. Baseline anatomical characteristics ${ }^{a}$ and procedural information ${ }^{\mathrm{b}}$

\begin{tabular}{|c|c|}
\hline \multicolumn{2}{|l|}{ Variable } \\
\hline No. of patients & 9 \\
\hline \multicolumn{2}{|l|}{ Anatomic characteristics ${ }^{a}$} \\
\hline Diameter at SMA, mm & $26(14.8-34)$ \\
\hline Diameter at highest RA, $\mathrm{mm}$ & $29.7(16.3-44.9)$ \\
\hline Diameter at lowest RA, $\mathrm{mm}$ & $32(19-42)$ \\
\hline Infrarenal angulation, ${ }^{\circ}$ & $19(8.4-41)$ \\
\hline Suprarenal angulation, ${ }^{\circ}$ & $31.7(14.5-40.4)$ \\
\hline Maximum AAA diameter, $\mathrm{mm}$ & 59 (51.7-106.5) \\
\hline \multicolumn{2}{|l|}{$\begin{array}{l}\text { Procedural information, } \\
\text { primary/revision }\end{array}$} \\
\hline \multicolumn{2}{|l|}{ No. of chimney grafts } \\
\hline I & $5 / 0$ \\
\hline II & $3 / 0$ \\
\hline III & $1 / 2$ \\
\hline $\begin{array}{l}\text { Endobag fill } \\
\text { volume, } \mathrm{mL}\end{array}$ & $\begin{array}{l}90(25-180) / \\
20(20-20)\end{array}$ \\
\hline $\begin{array}{l}\text { Endobag fill pressure, } \\
\mathrm{mm} \mathrm{Hg}\end{array}$ & $\begin{array}{r}197.5(180-250) / \\
233(216-250)\end{array}$ \\
\hline Technical success & $9 / 2$ \\
\hline \multicolumn{2}{|c|}{$\begin{array}{l}\text { AAA, Abdominal aortic aneurysm; RA, renal artery; SMA, superior } \\
\text { mesenteric artery. } \\
\text { Values are median (range). } \\
\text { a Aneurysm characteristics at primary chimney endovascular aneurysm } \\
\text { sealing configuration (chEVAS) treatment. } \\
\text { bProcedural information including primary and revision chEVAS } \\
\text { procedures. }\end{array}$} \\
\hline
\end{tabular}

acquired within 1 month. Data collection was approved by the local institutional review board. The patient data were previously included in the ASCEND registry. ${ }^{4}$ Table I shows the baseline anatomical characteristics and procedural information. Advanta V12 covered chimney stents (Maquet Getinge Group, Hudson, NH) were used in all patients. No additional (self-expanding) stents were placed in the transition zone to the native vessel. Technical success was achieved in all procedures.

Image acquisition. ECG-gated CTA scans were acquired with a 256-slice CT scanner (Brilliance iCT 256 scanner, Philips Healthcare, Eindhoven, the 

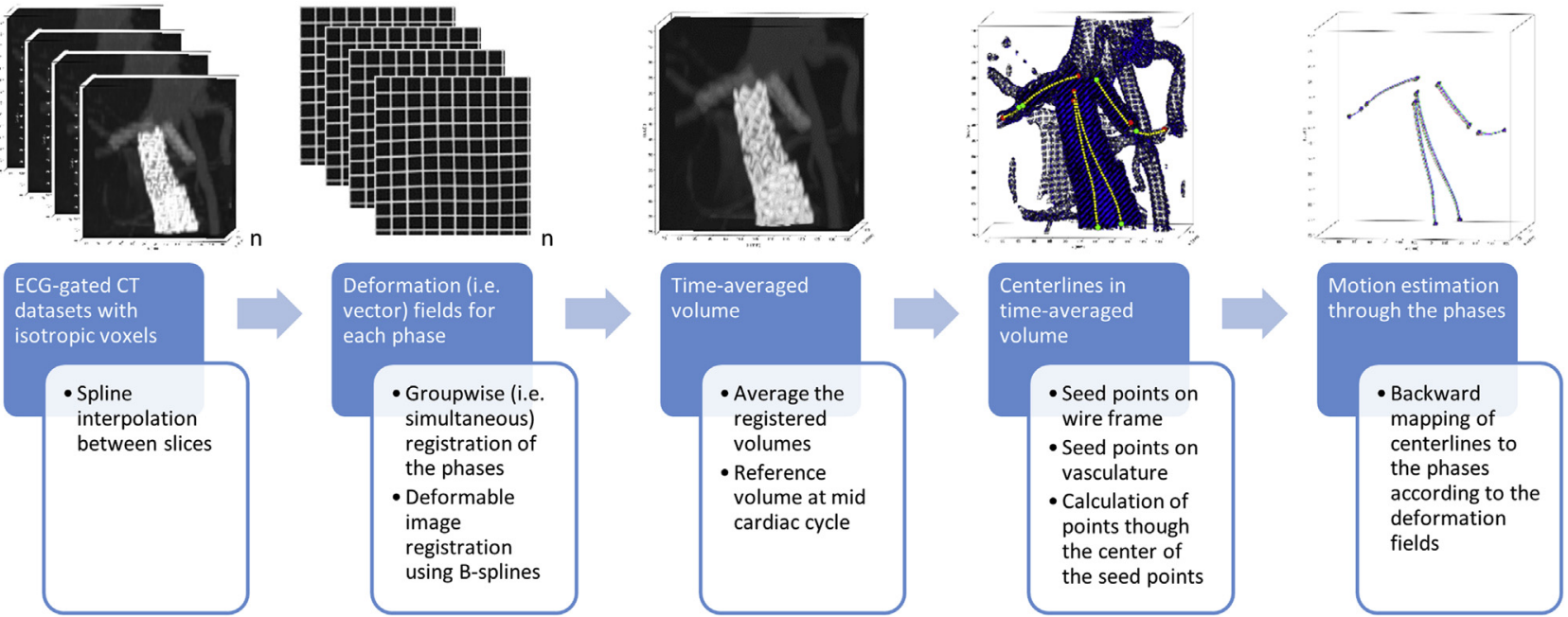

Fig 1. Flow chart showing the steps of the applied methodology for evaluation of 3-dimensional stent-graft motion in electrocardiogram (ECG)-gated computed tomography (CT).

Netherlands). Scanning parameters were similar to the conventional in-house used CTA scan protocol for EVAS follow-up and included a tube voltage of $100 \mathrm{kV}$, rotation time of 0.27 seconds, tube current time product of 63 to $236 \mathrm{mAs}$, collimation of $128 \times 0.625 \mathrm{~mm}$, pitch factor of 0.16 to 0.18 , and reconstructed matrix size of $512 \times 512$ pixels. Slice thickness was $2.5 \mathrm{~mm}$ with $2.5 \mathrm{~mm}$ spacing between slices (increment). Retrospective gating was performed at $10 \%$ intervals from $0 \%$ to $90 \%$ of the RR interval. For one dataset, an additional reconstruction was made with a slice thickness of $0.9 \mathrm{~mm}$ with $0.45-\mathrm{mm}$ increments. An intravascular contrast volume $(300 \mathrm{mg} \mathrm{l} / \mathrm{mL})$ of $80 \mathrm{~mL}$ was injected at a flow rate of $4 \mathrm{~mL} / \mathrm{s}$.

Image analysis. Image analysis was performed using a previously established combination of an image registration and a segmentation algorithm ${ }^{11}$ that was validated for motion estimation of endografts in ECG-gated CTA data. ${ }^{9}$ The algorithm was customized to quantify motion and deformation of the Nellix stents, the chimney grafts and the stented branch vessels during the cardiac cycle. An overview of the applied methodology is shown in Fig 1. First, the ECG-gated datasets with a slice thickness of $2.5 \mathrm{~mm}$ were interpolated in $z$-direction by spline interpolation to acquire submillimeter isotropic voxels. The interpolated volumes were registered simultaneously by a deformable (ie, elastic) registration algorithm dedicated to endograft deformation analysis. ${ }^{11}$ Elastic (ie, nonrigid) registration was necessary to allow for estimation of deformation, including bending of stents. The result of elastic registration is a deformation field for each reconstructed phase in the cardiac cycle, which can be used to compute the position of points in the data during the cardiac cycle. The deformation fields describe the displacement of all voxels with respect to the average of all phases, hereafter referred to as timeaveraged volume, which corresponds with the midcardiac cycle.

To analyze motion and changes in geometry, lumen centerlines of the stents and branch vessels were obtained, which run through the center of the stent's wire frame and the vessel lumen boundary, respectively (Fig 1). The wire frames were segmented as a set of points in the time-averaged volume by thresholding at 600 Hounsfield units. The vessel boundaries were obtained using an implementation of the marching cubes algorithm $^{12}$ in the open source functions of scikit-image ${ }^{13}$ v.O.11 at a threshold of 250 Hounsfield units. The vertices of the resulting surface mesh were used as vessel boundary points. Centerlines of the stents and branch vessels were extracted by calculating the path through the center of the respective point set by maximizing the distance to the point set. The step size of points along the centerline was set to $1 \mathrm{~mm}$. The deformation fields were applied to the centerlines by backward mapping to estimate the position of the centerlines at each phase in the cardiac cycle.

Finally, translation of the proximal segment of the Nellix stents and the proximal and distal segments of the chimney grafts; component stability expressed as distance change between the proximal ends of both Nellix stents (Nellix-to-Nellix distance) and between the proximal ends of the Nellix stents and the chimney grafts (Nellix-to-chimney distance); and angulation change of the chimney grafts themselves (chimney angle), between the proximal segment of the Nellix stents and the chimney grafts (Nellix-to-chimney angle), and between the distal segment of the chimney grafts and the branch vessel distal to the chimney grafts (end-stent angle) were quantified during the cardiac cycle (Fig 2). Segments of 10 centerline points $(10 \mathrm{~mm})$ were used. 

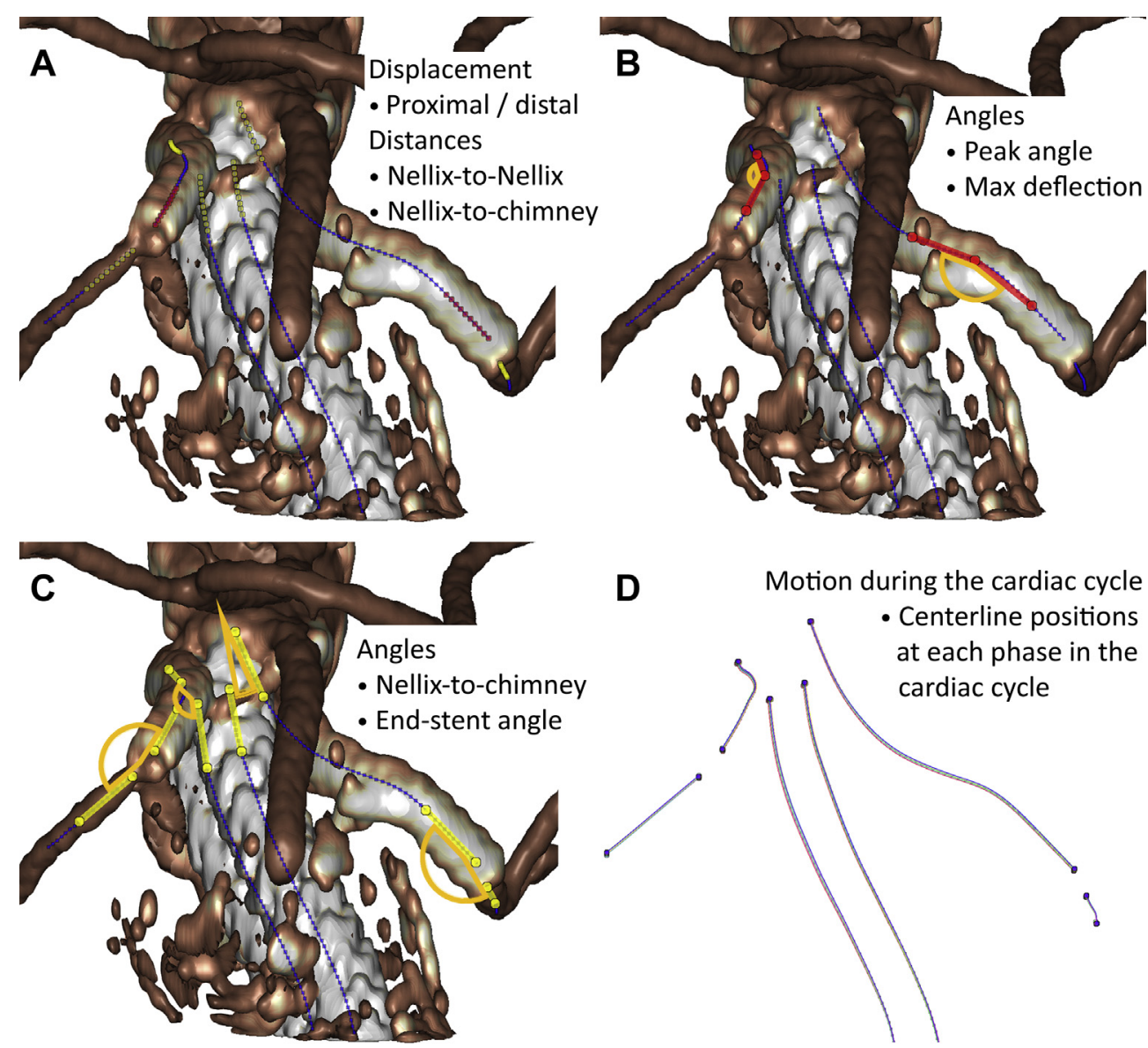

Fig 2. Dynamic computed tomography angiography (CTA) measurements of the Nellix stents, the chimney grafts, and the branch vessels distal to the chimney grafts. Measurements describe cardiac pulsatility-induced displacement of the Nellix stents and chimneys and distances from Nellix to Nellix and from Nellix to chimney (A), chimney angles defined as maximum deflection angles and peak angles (B), and angles from Nellix-tochimney and end-stent angles from chimney to branch vessel $\mathbf{( C )}$. The relative position of the centerlines during the cardiac cycle is shown for the case example (\#2) is presented (D). Minor displacement and deformation of the Nellix stents, the chimney grafts, and the branch vessel is seen during the cardiac cycle.

The chimney angles were calculated in a triangular orientation as the angle between two $10-\mathrm{mm}$ vectors from a point on the centerline to two other centerline points at a fixed arm length of $10 \mathrm{~mm}$. The maximum angulation change of the chimney graft during the cardiac cycle (chimney deflection) was identified at a certain point on the centerline (ie, the point at which the angulation change was greatest). In addition, the maximum angle of the centerline was assessed at each phase in the cardiac cycle to obtain change in maximum chimney angle (peak angle change). The Nellix-tochimney and end-stent angles were calculated as the angle between the directional vectors of the centerline segments. An angle of zero indicates a straight line.

For validation purposes, an additional analysis was performed on the $0.9 \mathrm{~mm}$-slice reconstruction that was available for one of the datasets. Interpolation between the slices was not applied to this additional dataset. A comparison of cyclic motion and deformation was made between the $0.9-\mathrm{mm}$ and $2.5-\mathrm{mm}$ slice reconstructions (Supplementary Material, online only).

Statistics. Descriptive data were summarized as mean \pm standard deviation (range). Categorical data were expressed as numbers. Comparative measurements were performed using an independent samples $t$-test. Statistical significance was assumed at a $P$ value of less than .05. The open source statistical functions of Scipy ${ }^{14}$ v.1.1 were used with Miniconda3 as Python interpreter (Python 3).

\section{RESULTS}

All image processing was successfully performed. Centerlines were obtained in all 11 ECG-gated CTA datasets, including 22 Nellix stents and 20 chimney grafts (left renal artery [LRA], $n=9$; right renal artery [RRA], $n=8$; and superior mesenteric artery [SMA], $n=3$ ). An example of the dynamic CTA analysis performed is shown in Fig 2. A maximum intensity projection cine-loop of the original 
Table II. Translation during the cardiac cycle of the Nellix stents and the chimney grafts

\begin{tabular}{|c|c|c|c|c|}
\hline & \multicolumn{4}{|c|}{ Magnitude of displacement, $\mathrm{mm}$} \\
\hline & $x$-Direction & $y$-Direction & z-Direction & Three dimensional \\
\hline \multicolumn{5}{|c|}{ Nellix proximal } \\
\hline Left & $0.5 \pm 0.2(0.2-0.9)$ & $0.5 \pm 0.2(0.2-1.0)$ & $0.6 \pm 0.3(0.2-1.2)$ & $0.9 \pm 0.4(0.4-1.5)$ \\
\hline Right & $0.5 \pm 0.3(0.1-0.9)$ & $0.5 \pm 0.2(0.2-0.9)$ & $0.6 \pm 0.3(0.1-1.2)$ & $1.0 \pm 0.4(0.3-1.7)$ \\
\hline Mean & $0.5 \pm 0.2(0.1-0.9)$ & $0.5 \pm 0.2(0.2-1.0)$ & $0.6 \pm 0.3(0.1-1.2)$ & $1.0 \pm 0.4(0.3-1.7)$ \\
\hline \multicolumn{5}{|c|}{ Chimney graft LRA } \\
\hline Proximal & $0.5 \pm 0.2(0.3-0.9)$ & $0.5 \pm 0.2(0.3-0.8)$ & $0.5 \pm 0.3(0.1-1.2)$ & $0.9 \pm 0.4(0.4-1.7)$ \\
\hline Distal & $0.5 \pm 0.2(0.2-0.7)$ & $0.5 \pm 0.2(0.2-0.7)$ & $0.6 \pm 0.3(0.1-1.2)$ & $0.8 \pm 0.3(0.4-1.5)$ \\
\hline \multicolumn{5}{|c|}{ Chimney graft RRA } \\
\hline Proximal & $0.5 \pm 0.2(0.2-0.8)$ & $0.6 \pm 0.2(0.3-1.0)$ & $0.5 \pm 0.2(0.1-1.0)$ & $0.9 \pm 0.4(0.5-1.6)$ \\
\hline Distal & $0.4 \pm 0.1(0.2-0.6)$ & $0.5 \pm 0.2(0.1-0.8)$ & $0.6 \pm 0.2(0.2-0.9)$ & $0.8 \pm 0.3(0.3-1.3)$ \\
\hline \multicolumn{5}{|c|}{ Chimney graft SMA } \\
\hline Proximal & $0.5 \pm 0.3(0.3-0.9)$ & $0.6 \pm 0.3(0.4-1.0)$ & $0.4 \pm 0.2(0.1-0.6)$ & $0.8 \pm 0.5(0.4-1.5)$ \\
\hline Distal & $0.5 \pm 0.3(0.2-0.8)$ & $0.6 \pm 0.3(0.1-0.8)$ & $0.4 \pm 0.3(0.2-0.8)$ & $0.8 \pm 0.5(0.3-1.4)$ \\
\hline \multicolumn{5}{|c|}{ Chimney graft mean } \\
\hline Proximal & $0.5 \pm 0.2(0.2-0.9)$ & $0.6 \pm 0.2(0.3-1.0)$ & $0.5 \pm 0.3(0.1-1.2)$ & $0.9 \pm 0.4(0.4-1.7)$ \\
\hline Distal & $0.5 \pm 0.2(0.2-0.8)$ & $0.5 \pm 0.2(0.1-0.8)$ & $0.5 \pm 0.3(0.1-1.2)$ & $0.8 \pm 0.3(0.3-1.5)$ \\
\hline
\end{tabular}

ECG-gated CT volumes is shown in Supplementary Video 1 (online only). The corresponding constructed centerline models are shown in Supplementary Video 2 (online only).

Device translation and component stability during the cardiac cycle. Translation during the cardiac cycle was quantified in the $x-, y$-, and $z$-directions, corresponding with the right-to-left, anterior-to-posterior, and cranialto-caudal direction, and in three-dimensional space, that is, the vector length. The mean displacement of the Nellix stents and the chimney grafts is shown in Table II. Overall, the three-dimensional displacement was at most $1.7 \mathrm{~mm}$ for both the Nellix stents and the chimney grafts, showing no statistically significant differences between the proximal and distal segment of the chimney grafts $(0.9 \pm$ $0.4 \mathrm{~mm}$ vs $0.8 \pm 0.3 \mathrm{~mm} ; P=.632$ ), and between the proximal segments of the Nellix stents and the chimney grafts $(1.0 \pm 0.4 \mathrm{~mm}$ vs $0.9 \pm 0.4 \mathrm{~mm} ; P=.540)$. Distances from Nellix-to-Nellix stents and from Nellix-to-chimney stents during the cardiac cycle are shown in Fig 3. The maximum change in distance was on average $0.2 \pm 0.1 \mathrm{~mm}$ (range, $0.1-0.4 \mathrm{~mm}$ ) from Nellix-to-Nellix and $0.2 \pm 0.1 \mathrm{~mm}$ (range, 0.0-0.4 mm) from Nellix to chimney, with no statistically significant difference $(P=.823)$. The maximum change in distance was greatest in the postchEVAS scan of a patient (patient 7), in whom stent migration with a type la endoleak was identified (Nellix to Nellix, $0.4 \mathrm{~mm}$; Nellix to chimney, $0.4 \mathrm{~mm}$ ).

Chimney angulation during the cardiac cycle. Bending of the chimney grafts themselves and the Nellix-to-chimney angulation during the cardiac cycle was quantified (Fig 4).
The chimney deflection angles of the LRA, RRA, and SMA varied on average by $1.1 \pm 0.4^{\circ}$ (range, $\left.0.7^{\circ}-1.9^{\circ}\right), 1.2 \pm 0.8^{\circ}$ (range, $0.6^{\circ}-2.4^{\circ}$ ), and $1.6 \pm 0.8^{\circ}$ (range, $0.5^{\circ}-2.6^{\circ}$ ), respectively. The deflection angles of the renal chimney grafts did not significantly differ from the SMA chimney grafts (range, $1.2 \pm 0.6^{\circ}$ vs $1.6 \pm 0.8^{\circ} ; P=.296$ ). Overall, the mean change in chimney deflection angle was $1.2 \pm 0.7^{\circ}$ (range, $0.5^{\circ}-2.6^{\circ}$ ). The average length of the chimney centerlines was $37.5 \pm$ $14.3 \mathrm{~mm}$ (range, 22-62 $\mathrm{mm}$ ). Deflection angles were located on average at $48 \pm 15 \%$ (range, $23 \%-80 \%$ ) of the chimney graft relative to the proximal end of the chimney.

The minimum and maximum peak angles of the LRA, RRA and the SMA chimney grafts were on average $21.0 \pm 11.2^{\circ}$ (range, $7.2^{\circ}-36.6^{\circ}$ ), $16.8 \pm 5.8^{\circ}$ (range, $2.4^{\circ}$ $\left.23.1^{\circ}\right)$, and $14.9 \pm 3.8^{\circ}\left(\right.$ range, $\left.10.1^{\circ}-19.5^{\circ}\right)$ versus $21.7 \pm$ $11.2^{\circ}$ (range, $7.9^{\circ}-37.4^{\circ}$ ), $17.7 \pm 5.8^{\circ}$ (range, $3.4^{\circ}-24.5^{\circ}$ ), and $15.7 \pm 4.0^{\circ}$ (range, $10.6^{\circ}-20.5^{\circ}$ ), respectively, with no significant differences between renal and SMA chimney grafts for both minimum $(P=.483)$ and maximum $(P=.481)$ peak angles. The absolute change in peak angle of the LRA, RRA and the SMA chimney grafts was on average $0.8 \pm 0.3^{\circ}$ (range, $0.3^{\circ}-1.5^{\circ}$ ), $0.9 \pm 0.4^{\circ}$ (range, $0.5^{\circ}-1.6^{\circ}$ ) and $0.8 \pm 0.2^{\circ}$ (range, $0.5^{\circ}-1.0^{\circ}$ ), respectively. At mid cardiac cycle, the LRA, RRA, and SMA peak angles were on average located at $57 \pm 10 \%$ (range, 44\%-68\%), $55 \pm$ $11 \%$ (range, $42 \%-76 \%$ ), and $44 \pm 1 \%$ (range, $43 \%-46 \%$ ) of the chimney graft relative to the proximal end of the chimney. The location of the peak angle during the cardiac cycle changed by $0.4 \pm 0.3 \mathrm{~mm}$ (range, 0.1-1.2 mm), $0.6 \pm 0.5 \mathrm{~mm}$ (range, 0.1-1.8 mm) and $1.3 \pm 1.1 \mathrm{~mm}$ (range, $0.1-2.7 \mathrm{~mm}$ ) for the LRA, RRA, and SMA, respectively. 

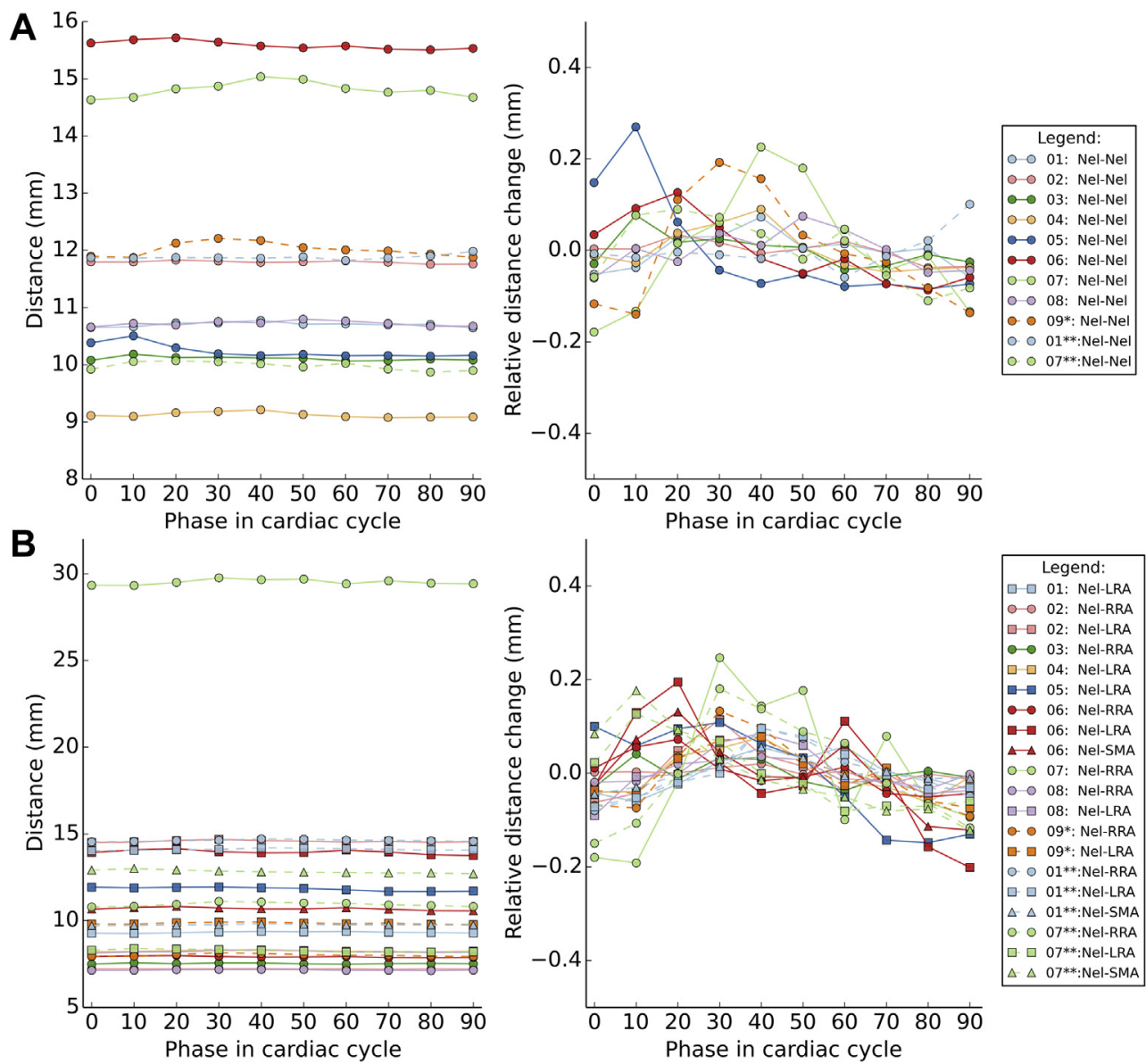

Fig 3. Distance change from Nellix to Nellix (A) and from Nellix to chimney (B) during the cardiac cycle. Absolute distances are shown (left) as well as the relative change in distance with respect to the distance at mid cardiac cycle (right). LRA, left renal artery; Nel, Nellix stent; Nel-Nel, Nellix to Nellix; RRA, right renal artery; SMA, superior mesenteric artery.

The Nellix-to-chimney angles for the LRA, RRA and SMA varied on average by respectively $0.7 \pm 0.3^{\circ}\left(0.4^{\circ}-1.3^{\circ}\right)$, $1.0 \pm 0.3^{\circ}$ (range, $0.5^{\circ}-1.7^{\circ}$ ), and $0.8 \pm 0.4^{\circ}$ (range, $0.3^{\circ}$ $\left.1.3^{\circ}\right)$ during the cardiac cycle. There was no significant difference between renal and SMA chimney grafts ( $0.9 \pm$ $0.3^{\circ}$ vs $0.8 \pm 0.4^{\circ} ; P=.767$ ). Minimum and maximum Nellix-to-chimney angles for the LRA, RRA and the SMA chimney grafts were on average $143.8 \pm 12.0^{\circ}$ (range, $\left.124.5^{\circ}-161.8^{\circ}\right), 134.6 \pm 16.6^{\circ}$ (range, $\left.101.3^{\circ}-156.3^{\circ}\right)$, and $150.8 \pm 10.1^{\circ}$ (range, $141.9^{\circ}-164.9^{\circ}$ ) versus $144.5 \pm 11.9^{\circ}$ (range, $125.4^{\circ}-162.3^{\circ}$ ), $135.6 \pm 16.7^{\circ}$ (range, 102.1 $1^{\circ}-157.2^{\circ}$ ), and $151.5 \pm 9.8^{\circ}$ (range, $142.7^{\circ}-165.2^{\circ}$ ), respectively. There were no significant differences between renal and SMA chimney grafts for both minimum $(P=.253)$ and maximum $(P=.253)$ Nellix-to-chimney angles.

End-stent angle during the cardiac cycle. The endstent angles relative to the distal native branch vessels were quantified (Fig 5). The end-stent angles for the LRA, RRA, and SMA varied on average by, respectively, $1.7 \pm 0.9^{\circ}\left(\right.$ range, $\left.0.5^{\circ}-3.3^{\circ}\right), 1.9 \pm 0.8^{\circ}\left(\right.$ range, $0.7^{\circ}-3.3^{\circ}$ ), and
$1.3 \pm 0.4^{\circ}\left(\right.$ range, $\left.0.7^{\circ}-1.6^{\circ}\right)$ during the cardiac cycle. The difference was not statistically significant between the renal arteries and the SMAs $\left(1.8 \pm 0.8^{\circ}\right.$ vs $1.3 \pm 0.4^{\circ} ; P=$ .316). Overall, the end-stent angles varied on average by $1.7 \pm 0.8^{\circ}$ (range, $0.5^{\circ}-3.3^{\circ}$ ). The end-stent angles exhibited significantly greater change during the cardiac cycle compared to the Nellix-to-chimney angles $\left(1.7 \pm 0.8^{\circ} \mathrm{vs}\right.$ $\left.0.8 \pm 0.3^{\circ} ; P<.001\right)$. Minimum and maximum end-stent angles for the LRA, RRA, and the SMA were on average $47.2 \pm 18.4^{\circ}$ (range, $16.1^{\circ}-81.2^{\circ}$ ), $61.9 \pm 13.5^{\circ}$ (range, $41.7^{\circ}$ $77.0^{\circ}$ ), and $19.6 \pm 3.8^{\circ}$ (range, $14.6^{\circ}-23.6^{\circ}$ ) versus $48.8 \pm$ $18.0^{\circ}$ (range, $19.4^{\circ}-81.7^{\circ}$ ), $63.8 \pm 13.3^{\circ}$ (range, $43.7^{\circ}-79.3^{\circ}$ ), and $20.9 \pm 3.7^{\circ}\left(\right.$ range, $\left.16.1^{\circ}-25.1^{\circ}\right)$. The renal end-stent angles were greater compared to the SMA end-stent angles for both the minimum $\left(54.1 \pm 17.9^{\circ}\right.$ vs $19.6 \pm 3.8^{\circ}$; $P=.006)$ and maximum (55.9 $\pm 17.6^{\circ}$ vs $20.9 \pm 3.7^{\circ}$; $P=.004)$ end-stent angles.

Comparison of a 2.5- and $0.9-\mathrm{mm}$ slice reconstruction. All measurements were repeated on the $0.9 \mathrm{~mm}$ slice reconstruction to compare with the analysis of the 

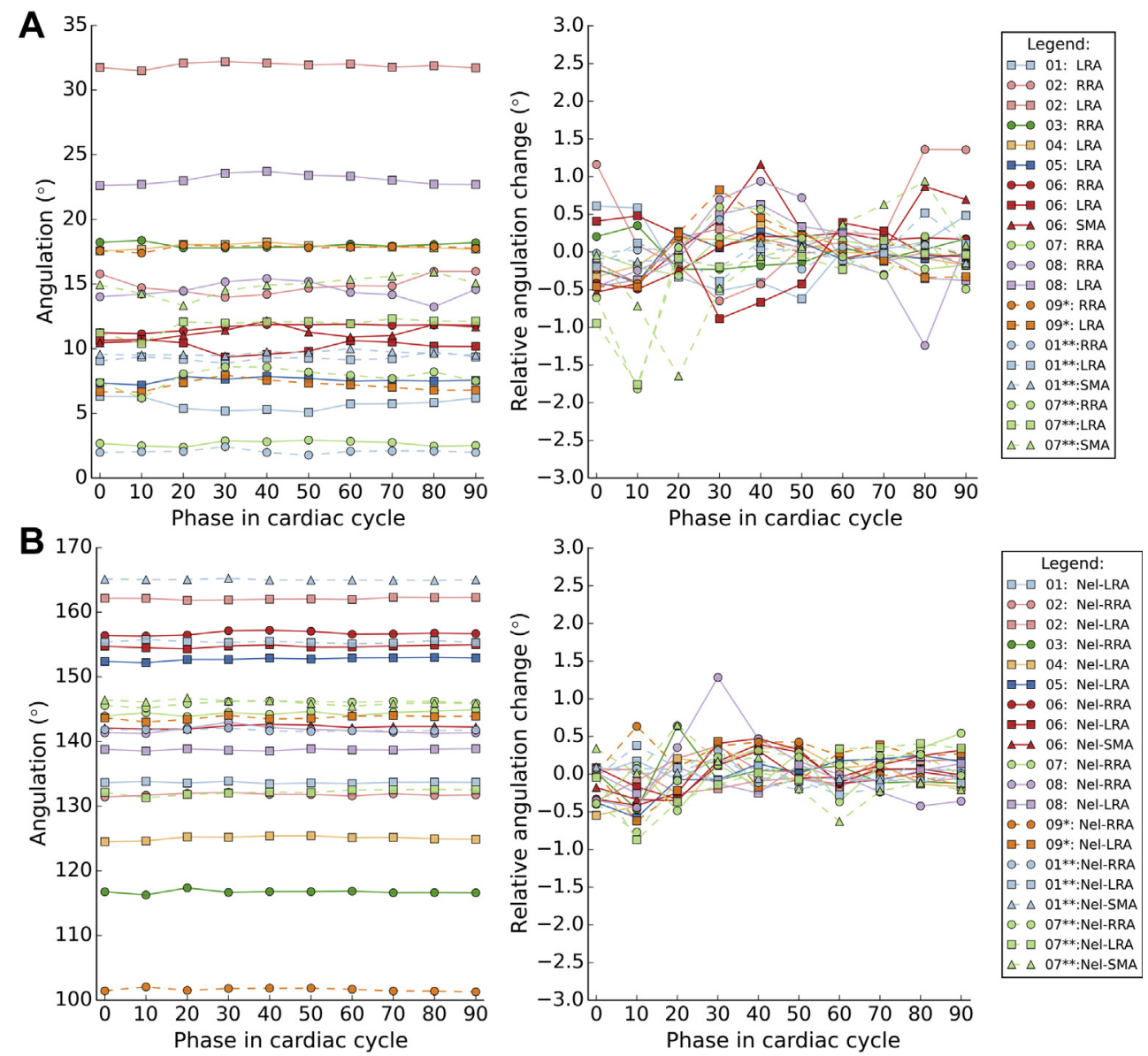

Fig 4. Bending of the chimney grafts during the cardiac cycle. Angles of maximum chimney deflection (A) and Nellix-to-chimney angles are displayed (B). Absolute angles are shown (left) as well as the relative change in angle with respect to the angle at mid cardiac cycle (right). LRA, left renal artery; Nel, Nellix stent; RRA, right renal artery; SMA, superior mesenteric artery.
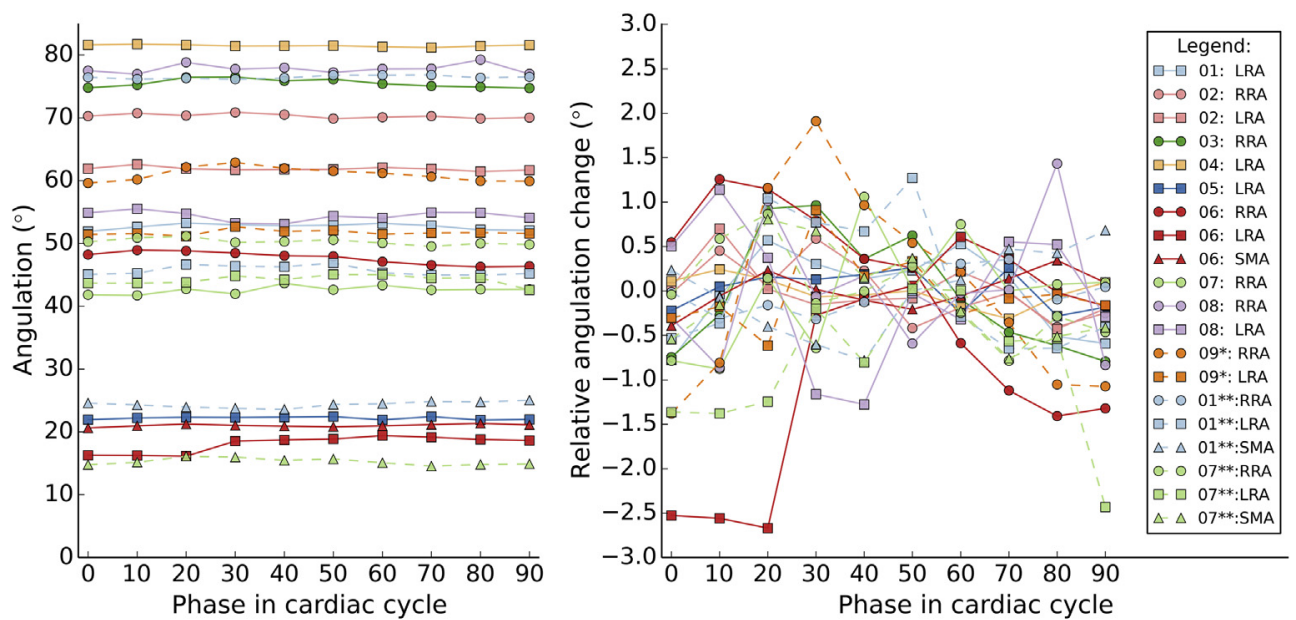

Fig 5. End-stent angle relative to the distal native branch vessel. Absolute angles are shown (left) as well as the relative change in angle with respect to the angle at mid cardiac cycle (right). LRA, left renal artery; RRA, right renal artery; SMA, superior mesenteric artery. 
$2.5 \mathrm{~mm}$ slice reconstruction. The highest deviations were $0.11 \mathrm{~mm}$ for the magnitude of displacement, $0.06 \mathrm{~mm}$ for distance change, $0.10^{\circ}$ for chimney deflection angle change, $0.25^{\circ}$ for the change in peak angle, $0.23^{\circ}$ for Nellix-to-chimney angle change, and $0.48^{\circ}$ for the endstent angle change. A comprehensive description of the comparison can be found in the Supplementary Material (online only).

\section{DISCUSSION}

The present study evaluated the cardiac pulsatilityinduced displacement and deformation of the proximal sealing and fixation zone of the Nellix EVAS System combined with chimney grafts. To our knowledge, this is the first study to perform such an analysis in multiphasic ECG-gated CTA data of patients treated with chEVAS. The proximal part of the Nellix stents and chimney grafts exhibited minimal displacement during the cardiac cycle (magnitude of displacement on average $\leq 1.0 \mathrm{~mm}$ ), with the device components moving together as a single unit.

It is important to emphasize that these results, although showing a stable situation, do not predict the potential for future migration, because other forces may contribute to this phenomenon. Lateral bending is considered as one of the driving factors behind caudal migration in EVAS, leading to type la endoleak. ${ }^{15}$ The stiffness of the stents and polymer-filled endobags seem to be key factors. This factor is reflected by the introduction of the thrombus index in the instructions for use. The thrombus index is defined as the ratio of the maximum AAA diameter and the maximum flow lumen diameter and should be less than 1.4. Moreover, it was recently advised to increase the rigidity of the system in case of Nellix-in-Nellix revision for distal migration, to minimize the risk of ongoing lateral bending of the stents. ${ }^{16,17}$ Little is known about the risk of migration after chEVAS. $^{4}$ In the present study sample, two patients required a chEVAS revision, one of whom presented with evident migration of the Nellix stents. Both patients were initially treated with a short seal zone $12 \mathrm{~mm}$ and $8 \mathrm{~mm}$ ). Two years after revision, both patients showed no signs of failure. ${ }^{10}$ This finding supports the analysis of the postrevision dynamic CTA scan performed in this study showing no deviant cyclic behavior from other chEVAS cases. Longitudinal studies using ECG-gated CTA scanning after chEVAS are indicated to further assess the predictive value of this scanning protocol. A progressive motion during the cardiac cycle might be an early indicator for decreased positional stability and as such the used scan technique could be of great value during follow-up of these and other complex procedures.

The Nellix-to-chimney angles were virtually constant during the cardiac cycle $\left(0.9 \pm 0.4^{\circ}\right)$ and the chimney grafts presented little bending deformation (1.2 $\left.\pm 0.7^{\circ}\right)$, which was supported by observing comparable translation at the proximal and distal ends of the chimney grafts. This may be favorable to prevent distortion or stent fractures by bending fatigue in the long term. From our present work, it seems that double and triple chimney configurations exhibit slightly greater chimney deflection angles compared with single chimney configurations (Fig 4), which may be related to the greater length of the chimney grafts that were used in the double and triple configurations compared to the single configurations ( $41.0 \pm 14.2 \mathrm{~mm}$ vs $27.0 \pm 7.5 \mathrm{~mm}$; $P=.065$ ); this may be important to consider for chimney standardization and device durability testing. These observations manifest chEVAS as a stable configuration during the cardiac cycle. However, it should be noted that the Advanta V12 balloon-expandable covered stent (Getinge, Wayne, NY) is a relatively stiff stent compared with a selfexpanding stent or a more flexible balloon expandable covered stent (eg, Viabahn VBX (W. L. Gore \& associates, Flagstaff, Ariz; BeGraft peripheral, Bentley Hechingen, Germany). Further research is necessary to investigate potential differences with other chimney graft configurations.

Our observations further show that the motion of the native side vessels is damped after chEVAS as preoperative renal artery movement has been reported to be in the order of 1 to $3 \mathrm{~mm} .^{18,19}$ Similarly, fenestrated endografts have been shown to limit side branch motion, which may be important with regard to branch vessel patency. ${ }^{18}$ It was previously shown by Itoga et $\mathrm{al}^{20}$ that, after EVAS, the Nellix stents undergo minimal pulsatile motion between mid-diastole and peak systole (magnitude of movement of $<0.5 \mathrm{~mm}$ ). In the present study, albeit still small, we observed fairly greater magnitudes of motion $(\leq 1.7 \mathrm{~mm}$ ). This finding might be explained by the transrenal positioning of the stents and thus the greater proximity of the stents to the heart compared to the infrarenal fixated stents in the study by Itoga et al. $^{20}$ Interestingly, because the Nellix stents were fixated infrarenally, this study further found a mechanical damping effect of the Nellix device on renal artery movement. This finding is in contrast with conventional transrenal or infrarenal endografts that do not seem to alter renal artery motion. ${ }^{18}$ The renal branch angle change owing to pulsatile motion reported in the case series by Itoga et $\mathrm{al}^{20}$ was similar to the Nellix-to-chimney angle change reported in the present study, indicating that the mechanical damping of renal artery movement is similar with EVAS and chEVAS, whereas a damping of SMA movement was observed with chEVAS only. In chEVAS, this damping effect could contribute to a more stable configuration and therewith lead to less stent migration.

Notably, compared with the Nellix-to-chimney angles, the end-stent angles relative to the distal native branch vessels exhibited considerable variation $\left(\leq 3.3^{\circ}\right)$. Although 
the inherent stiffness of the chimney stents limited cyclic chimney angulation, it may have induced an inflection point at the transition from chimney to vessel, as reflected by the more apparent end-stent angle. This may raise concerns regarding tissue irritation in the long term that might lead to stenosis or occlusion. ${ }^{19,21}$ Moreover, reintervention owing to renal occlusion or stenosis appears to be relatively common after chimney procedures. ${ }^{4,22}$ The ASCEND registry ${ }^{4}$ showed compression of the renal chimney grafts as the most prevalent cause of reintervention, although this concerned only 6 of 295 total patients. However, the follow-up period of the ASCEND registry is limited.

This study is limited in evaluating distinct postoperative scans only owing to the lack of preoperative dynamic imaging and longitudinal dynamic follow-up scans. However, the present study was not designed to evaluate the effect of endograft implantation on native vessel motion or to assess changes over time but instead to evaluate the proximal stability of the chEVAS configuration during the cardiac cycle. This study contributed to the understanding of in vivo pulsatile movement of the Nellix device, the chimney grafts and the branch vessels, which is essential in improving preclinical testing and future device design. Furthermore, we were limited by the data reconstructions that were available at $2.5-\mathrm{mm}$ slice reconstructions only, except for one dataset that, by its raw data, could also be reconstructed at $0.9-\mathrm{mm}$ slices. Although we interpolated between slices, the original slice thickness may have affected the accuracy of our measurements in $z$-direction. However, the cardiac pulsatility-induced motions were found to be similar in $x$ - and $y$-directions compared with the $z$-direction and comparison of the 0.9 - and $2.5-\mathrm{mm}$ slice reconstructions found minimal differences between the measurements. Another limitation of the study is the fact that all CT scans were acquired using a breath hold and, as a consequence, the potential impact of respiratory motion could not be studied.

The methodology applied in our present work uses an automated three-dimensional approach to assess motion throughout the reconstructed volumes that transcends previous two-dimensional approaches. Also, motion and deformation were assessed during the whole cardiac cycle rather than during two phases of the cardiac cycle only. Future studies using this methodology to investigate changes over time are awaited to evaluate device efficacy and durability of different endovascular procedures (eg, chimney, fenestrated, and branched configurations).

\section{CONCLUSIONS}

Only minimal cyclical changes in distance and angulation between the proximal chEVAS device components occur during the cardiac cycle. These observations manifest chEVAS as a stable configuration during the cardiac cycle. The chimney grafts themselves exhibited little cyclic bending, which may be favorable for preventing long-term metal fatigue. Still, the end-stent angles relative to the distal native branch vessels exhibited considerable variation, which may raise concerns regarding branch occlusion or stenosis. Longitudinal studies using ECG-gated CTA scanning after chEVAS are indicated to further assess the predictive value of this scanning protocol for positional stability and mechanical durability.

\section{AUTHOR CONTRIBUTIONS}

Conception and design: MK, ED, MR

Analysis and interpretation: MK, RG, MR

Data collection: MK, ED, ME, TL, PL, AK

Writing the article: MK

Critical revision of the article: MK, ED, ME, TL, PL, AK, RG, MR

Final approval of the article: MK, ED, ME, TL, PL, AK, RG, MR

Statistical analysis: MK

Obtained funding: MK, RG, MR

Overall responsibility: $\mathrm{MK}$

\section{REFERENCES}

1. De Bruin JL, Brownrigg JR, Patterson BO Karthikesalingam A, Holt PJ, Hinchliffe RJ, et al. The endovascular sealing device in combination with parallel grafts for treatment of juxta/suprarenal abdominal aortic aneurysms: short-term results of a novel alternative. Eur J Vasc Endovasc Surg 2016;52:458-65.

2. Roy IN, Charib M, Zerwes S, Jakob R, Torella F McWilliams RG, et al. Anatomical applicability of endovascular aneurysm sealing techniques in a consecutive cohort of fenestrated endovascular aneurysm repairs. J Endovasc Ther 2017;24:773-8.

3. Lindblad B, Bin Jabr A, Holst J, Malina M. Chimney grafts in aortic stent grafting: hazardous or useful technique? Systematic review of current data. Eur J Vasc Endovasc Surg 2015;50:722-31.

4. Thompson M, Youssef M, Jacob R, Zerwes S, Reijnen M, Szopinski P, et al. Early experience with endovascular aneurysm sealing in combination with parallel grafts for the treatment of complex abdominal aneurysms: the ASCEND registry. J Endovasc Ther 2017;24:764-72.

5. Boersen JT, Donselaar EJ, Groot Jebbink E, Starreveld R, Overeem SP, Slump CH, et al. Benchtop quantification of gutter formation and compression of chimney stent grafts in relation to renal flow in chimney endovascular aneurysm repair and endovascular aneurysm sealing configurations J Vasc Surg 2017;66:1565-73.

6. Paraskevas KI, Karouki M, Rehman A, Torella F McWilliams RG, England A, et al. Endovascular aneurysm sealing (EVAS) alone or in combination with chimney grafts (chEVAS) for treating complications of previous endovascular aneurysm repair (EVAR) Procedures. Cardiovasc Intervent Radiol 2018;41:1015-20.

7. Joseph SZ, Kwok CHR, Hockley JA, Garbowski MW Ferguson J, Samuelson S, et al. A technique of "chimney Nellix" for the management of type la endoleak after EVAR J Vasc Interv Radiol 2018;29:623-7. 
8. Verhoeven ELGG, Mani K. New technology failures: who to blame or time to be cautious? Eur J Vasc Endovasc Surg 2018;56:318-9.

9. Koenrades MA, Struijs EM, Klein A, Kuipers H, Reijnen MMPJ, Slump CH, et al. Quantitative stent-graft motion in ECGgated $\mathrm{CT}$ by image registration and segmentation: in vitro validation and preliminary clinical results. Eur J Vasc Endovasc Surg 2019;58:746-55.

10. van de Velde L, Zoethout AC, Lardenoije J-WHP, Reijnen MMPJ. Secondary endovascular aneurysm (EVAS) sealing in combination with chimney grafts to treat failed chimney EVAS. J Endovasc Ther 2019:26:265-8.

11. Klein A, Renema W, Vliet JA, Oostveen LJ, Hoogeveen Y, Schultze Kool LJ, et al. Motion calculations on stent grafts in AAA. In: Grundmann RT, editor. Diagnosis, Screening and treatment of abdominal, thoracoabdominal and thoracic aortic aneurysms. Rijeka (Croatia): InTechOpen; 2011. p. 125-44.

12. Lorensen WE, Cline HE. Marching cubes: a High resolution 3D surface construction algorithm. SIGGRAPH Comput Graph 1987;21:163-9.

13. van der Walt S, Schönberger JL, Nunez-Iglesias J, Boulogne F, Warner JD, Yager N, et al. scikit-image: image processing in Python. PeerJ 2014;2:e453.

14. Jones E, Oliphant T, Peterson P. SciPy: open source scientific tools for Python [Internet]. 2001. Availabe at: http://www. scipy.org/. Accessed August 19, 2019.

15. van den Ham LH, Holden A, Savlovskis J, Witterbottom A, Ouriel K, Reijnen MMPJ, et al. Editor's choice - occurrence and classification of proximal type I endoleaks after endovascular aneurysm sealing using the Nellix ${ }^{\mathrm{TM}}$ device. Eur J Vasc Endovasc Surg 2017:54:729-36.

16. Donselaar EJ, Holden A, Zoethout AC, Zeebregts CJ, Reijnen MMPJ. Feasibility and technical aspects of proximal Nellix-in-Nellix extension for late caudal endograft migration. J Endovasc Ther 2016:24:210-7.
17. Zoethout AC, Zerwes S, Zeebregts CJAM, Heyligers JMM, De Vries JPJM, Oberhuber A, et al. Preliminary outcome of Nellix-in-Nellix extensions in patients treated with failed endovascular aneurysm sealing. J Vasc Surg 2019;70: 1099-106.

18. Muhs BE, Vincken KL, Teutelink A, Verhoeven ELG, Prokop M, Moll FL, et al. Dynamic cine-computed tomography angiography imaging of standard and fenestrated endografts: differing effects on renal artery motion. Vasc Endovascular Surg 2008; 42:25-31.

19. Muhs BE, Teutelink A, Prokop M, Vincken KL, Moll FL, Verhagen HJM. Endovascular aneurysm repair alters renal artery movement: a preliminary evaluation using dynamic CTA. J Endovasc Ther 2006;13:476-80.

20. Itoga NK, Suh G-Y, Cheng CP. Stabilization of the abdominal aorta during the cardiac cycle with the sacanchoring Nellix device. Ann Vasc Surg 2018;52:312. e7-312.e12.

21. Ullery BW, Suh G-Y, Lee JT, Liu B, Stineman R, Dalman $\mathrm{RL}$, et al. Geometry and respiratory-induced deformation of abdominal branch vessels and stents after complex endovascular aneurysm repair. J Vasc Surg 2015;61:875-85.

22. Donas KP, Torsello GB, Piccoli G, Pitoulias GA, Torsello GF, Bisdas T, et al. The PROTAGORAS study to evaluate the performance of the Endurant stent graft for patients with pararenal pathologic processes treated by the chimney/snorkel endovascular technique. J Vasc Surg 2016;63:1-7.

Submitted Aug 19, 2019; accepted Jan 29, 2020.

Additional material for this article may be found online at www.jvascsurg.org. 


\section{APPENDIX (online only)}

\section{SUPPLEMENTARY MATERIAL}

comparison of a 2.5- and 0.9- $\mathrm{mm}$-slice reconstruction

This document provides a comparison of the dynamic computed tomography angiography measurements obtained from a 2.5- and 0.9- $\mathrm{mm}$-slice reconstruction of the dataset that was acquired for patient 1 after chimney endovascular aneurysm sealing revision with a triple chimney procedure.

Qualitative comparison of computed tomography data. Supplementary Fig 1 (online only) displays a qualitative comparison of a single volume obtained from the $2.5 \mathrm{~mm}$-slice (2.5-mm increment) reconstruction before and after interpolation in z-direction and a volume obtained from the $0.9 \mathrm{~mm}$-slice $(0.45-\mathrm{mm}$ increment) reconstruction without interpolation.

Quantitative comparison of measurements. A quantitative comparison was performed between measurements as obtained from the interpolated $2.5 \mathrm{~mm}$-slice reconstruction and the $0.9 \mathrm{~mm}$-slice reconstruction.

Displacement. Supplementary Fig 2 (online only) compares the $x-y-z$ displacement, corresponding with the right $(-)$ to left $(+)$, anterior $(-)$ to posterior $(+)$, and cranial $(-)$ to caudal $(+)$ directions, of the analyzed proximal and distal parts of the Nellix stents and chimney grafts. The magnitude of displacement (threedimensional) of the $2.5-\mathrm{mm}$ slice reconstruction deviated on average from the $0.9-\mathrm{mm}$ slice reconstruction by $0.05 \pm 0.03 \mathrm{~mm}$ and $0.05 \pm 0.04 \mathrm{~mm}$ for the Nellix stents and the chimney grafts, respectively.

Distance change. The cyclic distance change from Nellix-to-Nellix and from Nellix-to-chimney was compared and is displayed in Supplementary Fig 3 (online only). The distance change deviated by 0.06 $\mathrm{mm}$ from Nellix-to-Nellix and on average by $0.03 \pm 0.0$ $\mathrm{mm}$ from Nellix-to-chimney.

Chimney deflection angle, chimney peak angle, and Nellix-to-chimney angle. Chimney deflection angles and Nellix-to-chimney angles of the two reconstructions are shown in Supplementary Fig 4 (online only). The deflection angle change deviated on average by $0.05 \pm 0.04^{\circ}$. Peak angle change of the chimney grafts deviated on average by $0.13 \pm 0.09^{\circ}$. The Nellix-to-chimney angle change deviated by $0.16 \pm 0.04^{\circ}$.

End stent angle. Supplementary Fig 5 (online only) compares the end stent angles relative to the distal native branch vessels. The change in the end stent angle deviated on average by $0.28 \pm 0.17^{\circ}$.

Discussion. Minimal differences were found between the measurements performed on the $0.9-\mathrm{mm}$ slice and the interpolated $2.5-\mathrm{mm}$ slice reconstruction. The only notable difference was found in the absolute values of the left renal artery and the right renal artery maximum chimney deflection angles, which could be explained by a different location of the detected maximum deflection angle (left renal artery, at $25 \%$ vs $33 \%$, right renal artery, at $29 \%$ vs $70 \%$ from the proximal end of the chimney graft). Most likely, small differences in the course and the displacement of the centerline have resulted in different locations of maximum chimney graft deflection. Nevertheless, the change in deflection angle during the cardiac cycle was similar, resulting in no discrepancy in the results. Even though we were only able to compare measurements for one dataset, the present comparison provides confidence that in this study the $2.5-\mathrm{mm}$ slice reconstructions provide reliable results. 
A

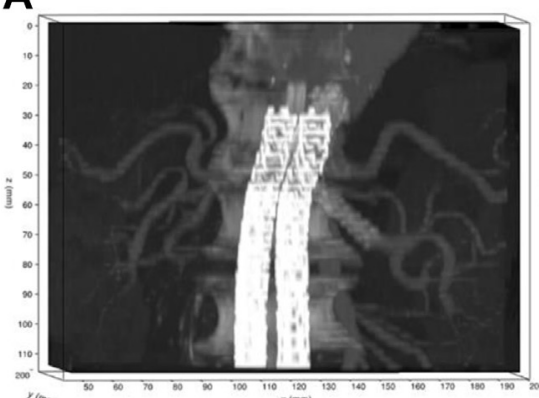

B

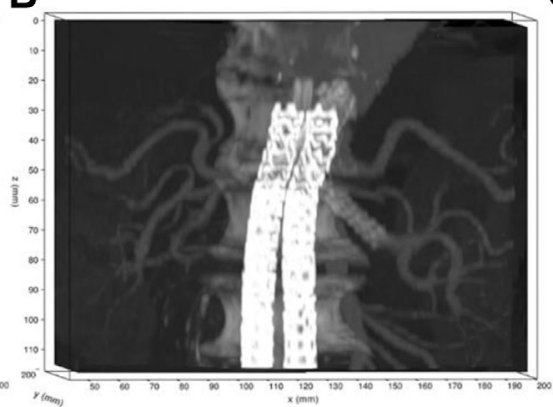

C

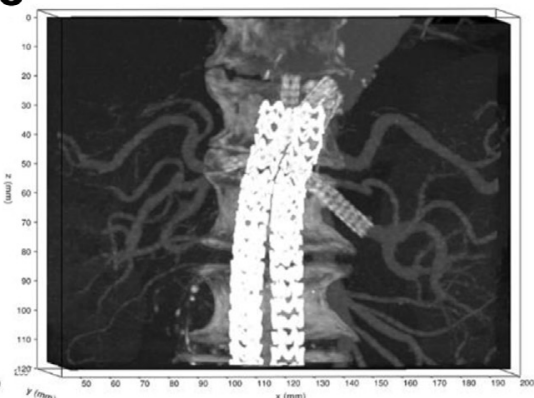

Supplementary Fig 1 (online only). Maximum intensity projections of a single volume at $20 \%$ of the cardiac cycle displaying the volume of the $2.5 \mathrm{~mm}$-slice reconstruction before (A) and after (B) interpolation and the volume of the $0.9-\mathrm{mm}$ slice reconstruction without interpolation (C).
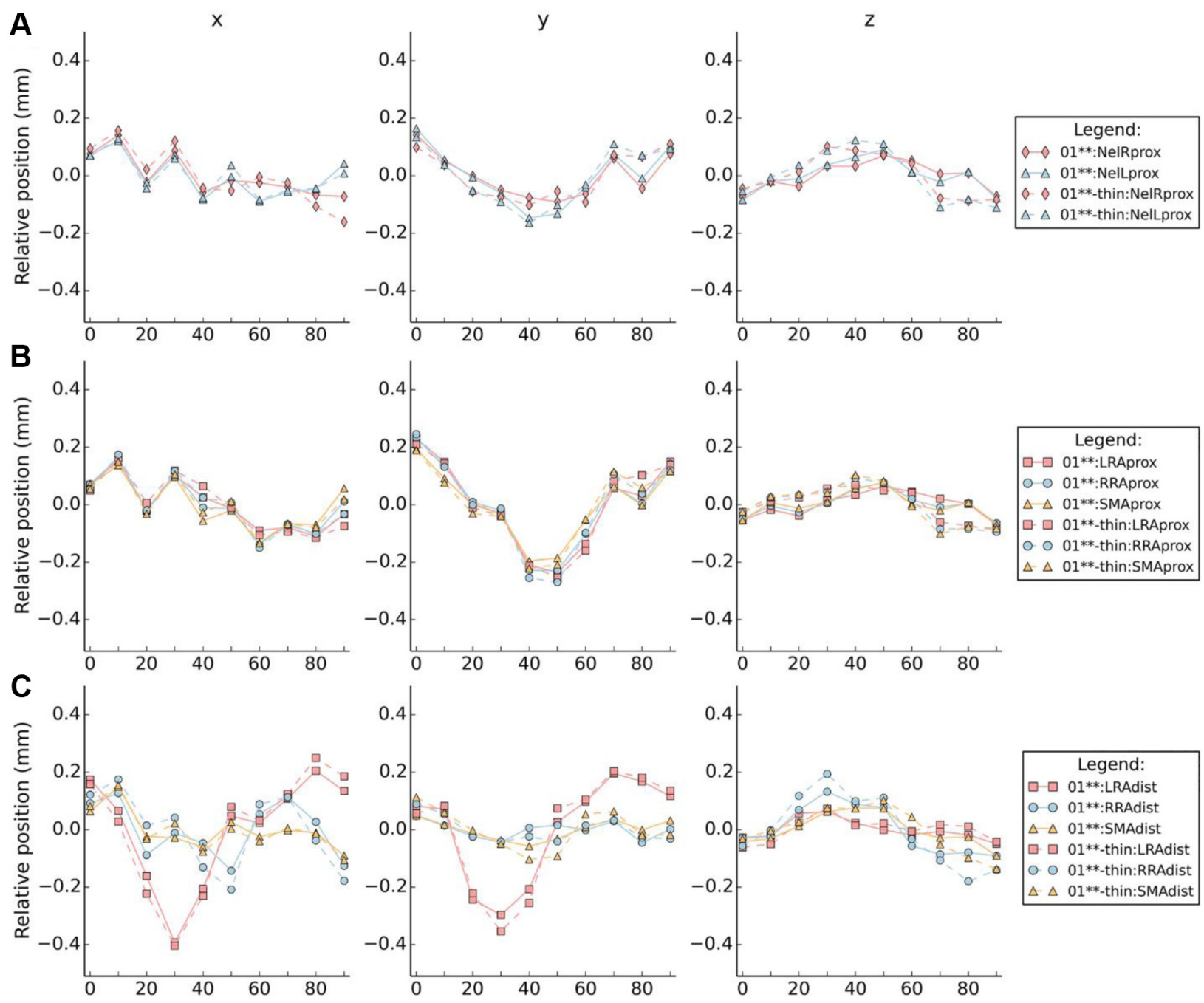

Phase in cardiac cycle

Phase in cardiac cycle

Phase in cardiac cycle

Supplementary Fig 2 (online only). Displacement in the $x$-, $y$-, and $z$-directions of the proximal (A and $\mathbf{B}$ ) and distal parts (C) of the Nellix stents and chimney grafts as measured in the $2.5-\mathrm{mm}$ (solid line) and 0.9-mm (dashed line) slice reconstruction. dist, distal; $L R A$, left renal artery; RRA, right renal artery; prox, proximal; SMA, superior mesenteric artery. 
Journal of Vascular Surgery Volume $\mathbf{m}$, Number
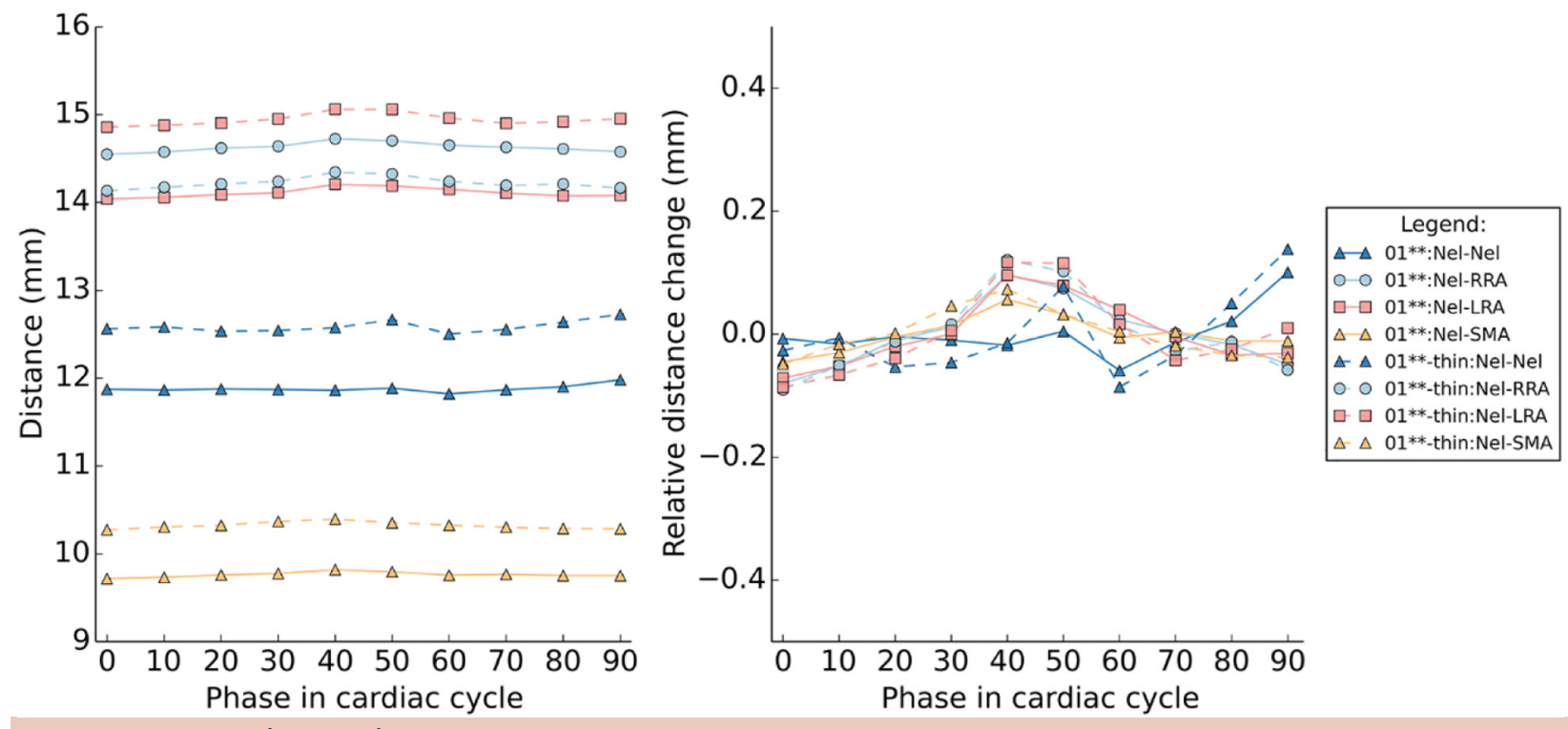

Supplementary Fig 3 (online only). Distance from Nellix-to-Nellix and from Nellix-to-chimney as measured in the 2.5-mm (solid line) and 0.9- $\mathrm{mm}$ (dashed line) slice reconstruction. LRA, left renal artery; Nel, Nellix stent; Nel-Nel, Nellix- to-Nellix; RRA, right renal artery; SMA, superior mesenteric artery. 

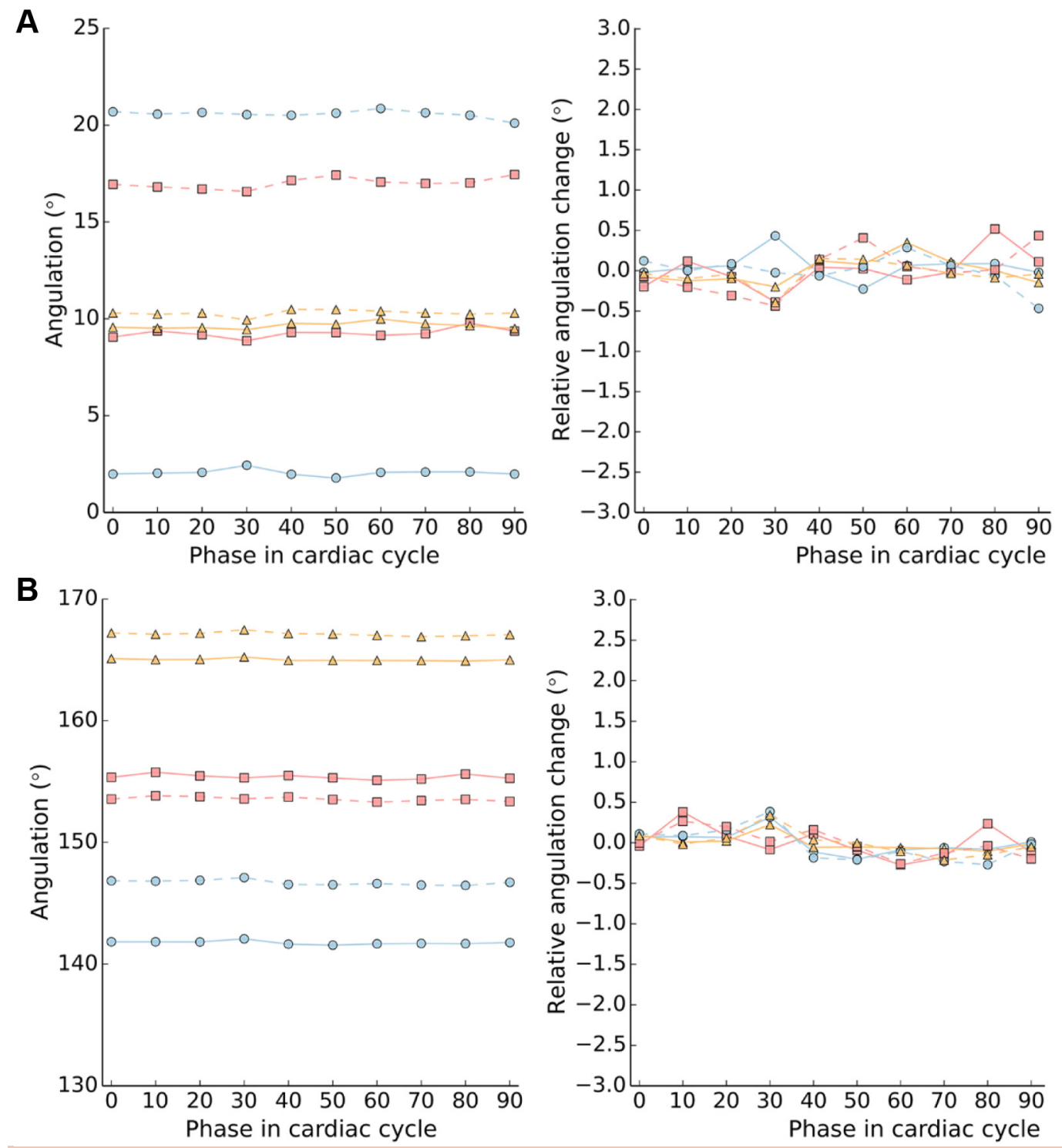

Supplementary Fig 4 (online only). Angles of maximum chimney deflection (A) and Nellix-to-chimney angles are displayed $(\mathbf{B})$ as measured in the $2.5-\mathrm{mm}$ (solid line) and $0.9-\mathrm{mm}$ (dashed line) slice reconstruction. $L R A$, left renal artery; Nel, Nellix stent; RRA, right renal artery; SMA, superior mesenteric artery. 

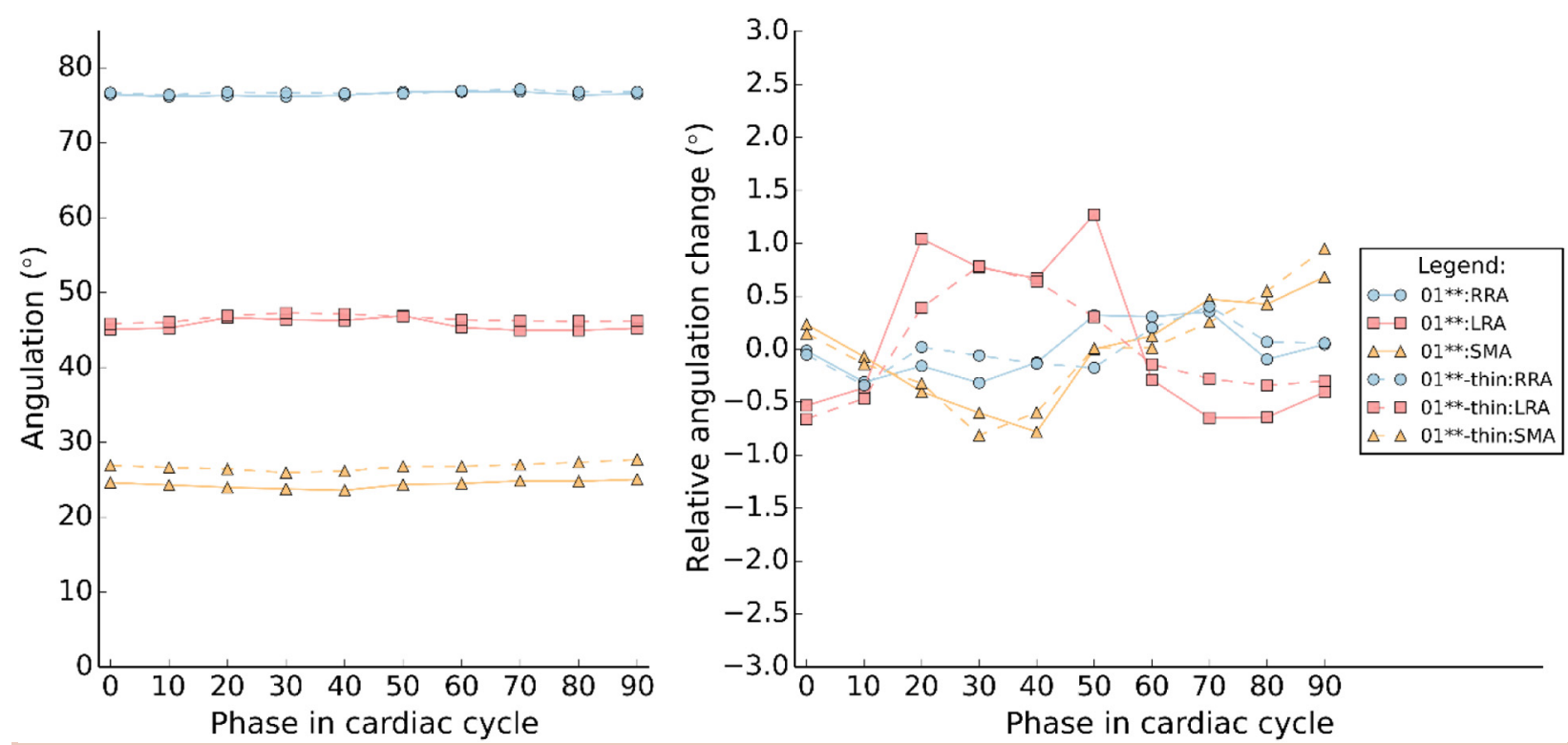

Supplementary Fig 5 (online only). End-stent angles as measured in the 2.5- $\mathrm{mm}$ (solid line) and 0.9-mm (dashed line) slice reconstruction. $L R A$, left renal artery; RRA, right renal artery; SMA, superior mesenteric artery. 